\title{
Neuromorphic tactile sensor array based on fiber Bragg gratings to encode object qualities
}

Sahana Prasanna, Luca Massari, Edoardo Sinibaldi, Renaud Detry, Joseph Bowkett, et al.

Sahana Prasanna, Luca Massari, Edoardo Sinibaldi, Renaud Detry, Joseph Bowkett, Kalind Carpenter, Calogero Maria Oddo, "Neuromorphic tactile sensor array based on fiber Bragg gratings to encode object qualities," Proc. SPIE 11136, Optics and Photonics for Information Processing XIII, 1113608 (6 September 2019); doi: 10.1117/12.2530733

EDent: SPIE Optical Engineering + Applications, 2019, San Diego, California, United States 


\title{
Neuromorphic tactile sensor array based on Fiber Bragg Gratings to encode object qualities
}

\author{
Sahana Prasanna ${ }^{\mathrm{a}}$, Luca Massari ${ }^{\mathrm{a}, \mathrm{b}}$, Edoardo Sinibaldi ${ }^{\mathrm{c}}$, Renaud Detry ${ }^{\mathrm{d}}$, Joseph Bowkett ${ }^{\mathrm{e}}$, Kalind \\ Carpenter ${ }^{\mathrm{d} *}$, Calogero Maria Oddo ${ }^{\mathrm{a} *}$
}

${ }^{a}$ The BioRobotics Institute, Scuola Superiore Sant'Anna, Viale Rinaldo Piaggio 34, 56025

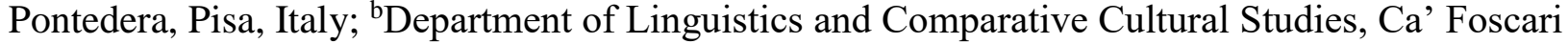
University of Venice, 30123 Venice, Italy; ${ }^{\mathrm{C}}$ Center for Micro-BioRobotics, Istituto Italiano di Tecnologia, Viale Rinaldo Piaggio 34, 56025 Pontedera, Pisa, Italy; 'det Propulsion Laboratory, NASA, 4800 Oak Grove Drive, 91109 Pasadena, California, USA; ${ }^{\mathrm{e} D e p a r t m e n t ~ o f ~ M e c h a n i c a l ~}$ \&amp; Civil Engineering, California Institute of Technology, 1200 East California Boulevard, 91125 Pasadena, California, USA

* These authors share senior authorship.

\begin{abstract}
Emulating the sense of touch is fundamental to endow robotic systems with perception abilities. This work presents an unprecedented mechanoreceptor-like neuromorphic tactile sensor implemented with fiber optic sensing technologies. A robotic gripper was sensorized using soft and flexible tactile sensors based on Fiber Bragg Grating (FBG) transducers and a neuro-bio-inspired model to extract tactile features. The FBGs connected to the neuron model emulated biological mechanoreceptors in encoding tactile information by means of spikes. This conversion of inflowing tactile information into event-based spikes has an advantage of reduced bandwidth requirements to allow communication between sensing and computational subsystems of robots. The outputs of the sensor were converted into spiking on-off events by means of an architecture implemented in a Field Programmable Gate Array (FPGA) and applied to robotic manipulation tasks to evaluate the effectiveness of such information encoding strategy. Different tasks were performed with the objective to grant fine manipulation abilities using the features extracted from the grasped objects (i.e., size and hardness). This is envisioned to be a futuristic sensor technology combining two promising technologies: optical and neuromorphic sensing.
\end{abstract}

Keywords: Neuromorphic, Fiber Bragg Grating, Tactile Soft sensor, FPGA neuron model, Izhikevich

\section{INTRODUCTION}

Neuromorphic computing permits to process a massive amount of multisensory data with low power consumption, high scalability and reduced data bandwidth [1]. Neuromorphic information processing takes inspiration from the biological neuronal systems where one hundred billions of biological neurons are connected, through thousands of synapses that form local memories, to process the sensory information in real time to provide unrivaled perceptual cognition [2].

Neuromorphic systems aim at mimicking the structural and functional characteristics of the neuronal networks using asynchronous event-driven spiking networks, and several biological systems provided a relevant source of inspiration, including the retinal [3], cochlear [4] and mechanoreceptive [5,6] neuronal architecture. Along this research stream, we addressed tactile neuromorphic systems with applications towards neuro-prosthetics [5], neurorobotics [7] and telepresence [8]. Indeed, tactile information is mediated by four types of functional units present in the cutaneous tissue (Merkel and Ruffini, which are slowly adapting, and Meissner and Pacinian, fast adapting), and human fingertips are innervated by largest amount of these mechanoreceptor organs extending into nerve fibers, which carry spiking information corresponding to tactile stimuli experienced by their respective receptive fields [9].

Various technologies are exploited to realize tactile transduction based on capacitive sensors [10], piezoelectric [11], piezoresistive [5]. The robotic community has taken many structural and functional inspirations to mimic the mechanics

Optics and Photonics for Information Processing XIII, edited by Khan M. Iftekharuddin,

Abdul A.S. Awwal, Victor H. Diaz-Ramirez, Andrés Márquez, Proc. of SPIE Vol. 11136

1113608 - @ 2019 SPIE · CCC code: 0277-786X/19/\$21 · doi: 10.1117/12.2530733 
the human hand $[12,13,14]$ according to individual focused application. Recent advancements towards the human-machine interactions has seen a boom in robotic manipulators [15] with human-like perception.

In this work, an under-actuated four-fingered hand (Cam-hand) of the JPL's four-legged RoboSimian robot with versatile mobility and manipulation capabilities [16] was used to perform grasping tasks. The complexity of tactile perception is being studied for years-long research [17,9,5]. This paper extends towards emulation of such a study with an upcoming tactile transduction technology of optical fibers. This technology offers various advantages in terms of high sensitivity, flexibility, electromagnetic immunity, multiplexing and scalable capabilities $[18,19]$. For the purpose of the tactile sensing, the Fiber Bragg Gratings (FBG) were chosen for their ability to measure and localize the applied strain [20,21]. Here, the contribution of temperature is negligible, since the whole experimental session was performed at room temperature.

Neuromorphic tactile encoding is a powerful tool to facilitate neuroscientists to study biological models through a robotic environment [22,5], which in turn can also be used to develop human-like manipulative intelligence for robots [6,23]. Here we present a novel tactile sensor aimed at providing relevant spatiotemporal spiking information as for first-order neurons [17] complementing neuromorphic chips inspired by brain networks. In particular, the proposed sensor is based on Fiber Bragg Grating (FBG) transducers [24] coupled to an Izhikevich neuron model [25]. Building on a previous implementation [20], the proposed sensor was integrated in a robotic gripper and experimentally validated by discriminating the stiffness of grasped samples made of different materials. We propose a novel neuromorphic FBG-based tactile system that is flexible, sensitive and easily adaptable for different platforms and applications.

\section{MATERIALS AND METHODS}

\subsection{Experimental setup and protocol}

The JPL's quadruped RoboSimian robot is equipped with an under-actuated hand (Cam-Hand) with manipulation and versatile mobility capabilities [16]. The hand consists of four aluminum fingers that were equipped with two optical fibers (running along the length of the finger) encased in a soft polymeric material in our previous work [20]. Each fiber hosted 6 FBGs. Each FBG has a microstructure etched periodically over few millimeters, along the length of optical fiber. Sensitivity to strain and temperature is obtained due to a change in period of these microstructures [26]. More in detail, the change in grating spatial period $\left(\Lambda_{B}\right)$ is given by $\lambda_{B}=2$. $\eta_{\text {eff. }} \Lambda_{B}$, where $\lambda_{B}$ denotes the reflected band of wavelength (Bragg wavelength), and $\eta_{\text {eff }}$ represents the effective refraction index. The chosen FBG technology can be easily multiplexed (inside a single optical fiber, each grating has unique $\lambda_{B}$ well separated from the others) and multiplexed FBGs provide multiple sensor sites, thus increasing sensor resolution.

Figure 1 illustrates the working concept through a parallel in between the proposed neuromorphic sensor [20] encoding and human touch [9]. Experimental validation was performed by grasping cylindrical samples (30mm diameter, $150 \mathrm{~mm}$ height). Four materials were considered, namely Dragon Skin (Young modulus $\mathrm{E} \approx 0.34 \mathrm{MPa})$, Vytaflex $(\mathrm{E} \approx 2 \mathrm{MPa}$ ), ABS $(\mathrm{E} \approx 2.2 \mathrm{GPa})$ and Aluminum $(\mathrm{E} \approx 70 \mathrm{GPa})$ with the purpose to categorize their stiffness. Each grasping task was repeated 10 times; fingers rotation was stopped when reaching a given threshold $(0.01 \mathrm{~nm})$ on wavelength variation. Data were recorded at $1 \mathrm{kHz}$ and processed as reported in the following. 


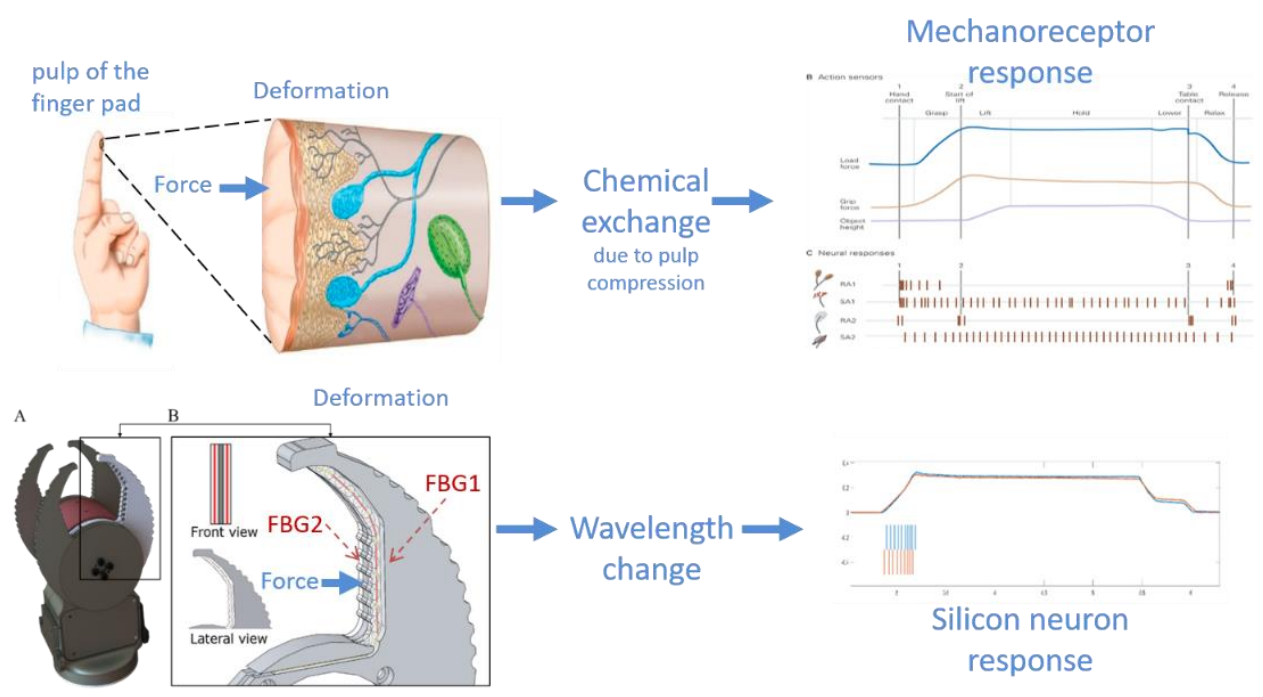

Figure 1 Neuromorphic FBG-based tactile sensor inspired by human mechanoreceptors. Top: The applied force on the human skin opens ionic channels of mechanoreceptors producing the chemical exchanges that lead the neurons to spike. Bottom: The applied force on the FBG changes the wavelength of the reflected light which then acts as the input current to an artificial neuron model producing correponding spikes

\subsection{Neuromorphic model}

In this study, we implemented and evaluated a model emulating fast adapting mechanoreceptors (RA1) to investigate its stiffness encoding capacity during the loading phase with constant velocity. RA1 receptors are responsive to encode the interaction with the skin during the dynamic phases of the grasp, unlike SA1 units that are more sensitive not only during the loading phase, but also during the static holding phase [27]. Two FBGs (FBG1 and FBG2 in Figure 1), each one integrated in optical fibers along the longitudinal axis of the finger, were chosen to mimic these receptors through neuromorphic processing. The raw sensor signals, from each FBG, were cut into an observational window that included the data points of the loading phase for each trial. The pre-processing of the windowed sensor data $(\Delta \lambda)$ was carried out by first-order bandpass IIR filter (forward coefficients: [0.045, 0, -0.045]; reverse coefficients: [1,-1.919,0.91]). The filtered output $\left(I_{\text {filt }}\right)$, with an amplification factor of 180 , was fed as current value into the following differential equation (Izhikevich neuron model):

$$
\begin{aligned}
& \frac{d v}{d t}=A v^{2}+B v+C-u+S I_{f i l t} \\
& \frac{d u}{d t}=a(b v-u)
\end{aligned}
$$

where $v$ is the membrane potential, $u$ is the membrane recovery variable and the constants for the model: $\mathrm{A}=0.04 \mathrm{~ms}^{-1} \mathrm{mV}^{-1}$; $\mathrm{B}=5 \mathrm{~ms}^{-1} ; \mathrm{C}=140 \mathrm{mVms}^{-1} ; \mathrm{a}=0.02 \mathrm{~ms}^{-1} \mathrm{~b}=0.2 \mathrm{~ms}^{-1} ; \mathrm{c}=-65 \mathrm{mV} ; \mathrm{d}=8 \mathrm{mV} ; \mathrm{Vth}=30 \mathrm{mV} ; \mathrm{k}=10 \mathrm{mVms}^{-1} \mathrm{~N}^{-1} 157 ; \mathrm{dt}=0.0625 \mathrm{~ms}$; $\mathrm{S}=180$. The value of $\mathrm{S}$ was so chosen that all the neurons spike for every trial while staying below the saturation of the neuron model during rest phases. All the other constants are the same as our previous works [8]. Two replicas of this neuron model (N1 and N2 in Figure 6) were chosen to independently provide inputs to the currents, $I_{\text {filt }}$, from two FBGs. The stiffness information from the neuronal model was evaluated in terms of the inter-spike-intervals (ISI) of the spikes produced, with stiffer materials we expect lower ISI (thus, higher firing rate). The obtained ISI was classified and tested using the Classification Learner app [28] provided by Mathworks to estimate the potential accuracy. A supervised learning algorithm called decision trees [29] was used to obtain the classification for this work. In particular, the coarse decision tree was used to build the model using the training set, Figure 2, while evaluating the model with the test set. The division between the training and test sets was performed by Matlab using a 50-fold cross validation. Cross-validation helps in utilizing the small dataset for both training and testing without overfitting the model. 


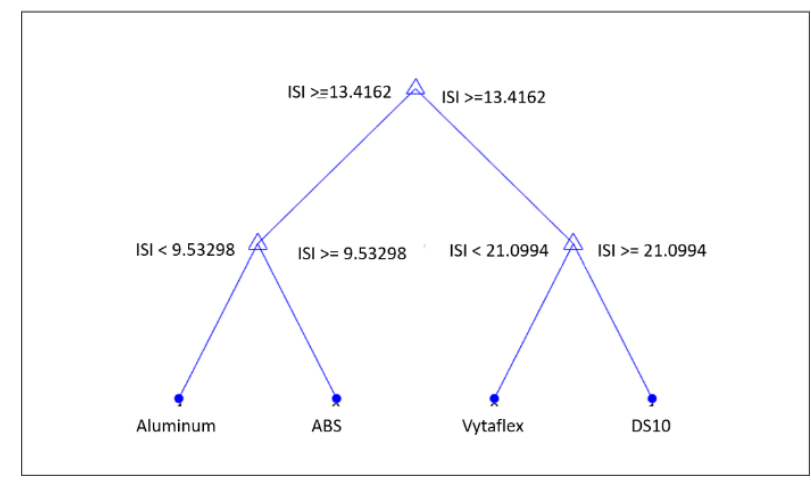

Figure 2 The classification tree

\subsection{Neuronal FPGA architecture}

The above described Izhikevich neuron model was also implemented on a Xilinx FPGA board provided in NI-sbRIO 9636. The FPGA neuron-computational core updates the membrane potential parameters with the proposed parallel and pipelined topology as shown in Figure 3. The computational units between the dotted vertical lines are executed in one-time cycle of the FPGA, i.e. $25 \mathrm{~ns}$. These computations use the fixed-point architecture to optimize resource consumption, the 18 bits representation, bit representation shown in red as 'integer part. decimal part'), around the multipliers are motivated by the available $18 \times 18$ bits multipliers, while leaving more resolution for adders and subtractors. Our design operates at $40 \mathrm{MHz}$ while each neuron updates at $16 \mathrm{kHz}$, leaving space for 2500 neurons on the same core. The same design was also tested to work at $70 \mathrm{MHz}$, with 4375 neuron updates.

The architecture of the ODE is divided into five pipelines considering execution time for each set of computation not exceeding $14 \mathrm{~ns}$. In the first pass, the neuron parameters are updated after 5 clock cycles, while the subsequent updates happen every cycle. Thanks to the pipeline, the output from one stage (represented in different colors) becomes the input for the next stages in the following clock cycle, Figure 4. The validation of spike trains, generated by one neuron, was performed against the computer simulated, in Matlab, Izhikevich ODE with $\mathrm{dt}=1 / 16$ executed 4 times on the same value of I to facilitate the benchmarking of the real-time and FPGA combination in the proposed architecture.

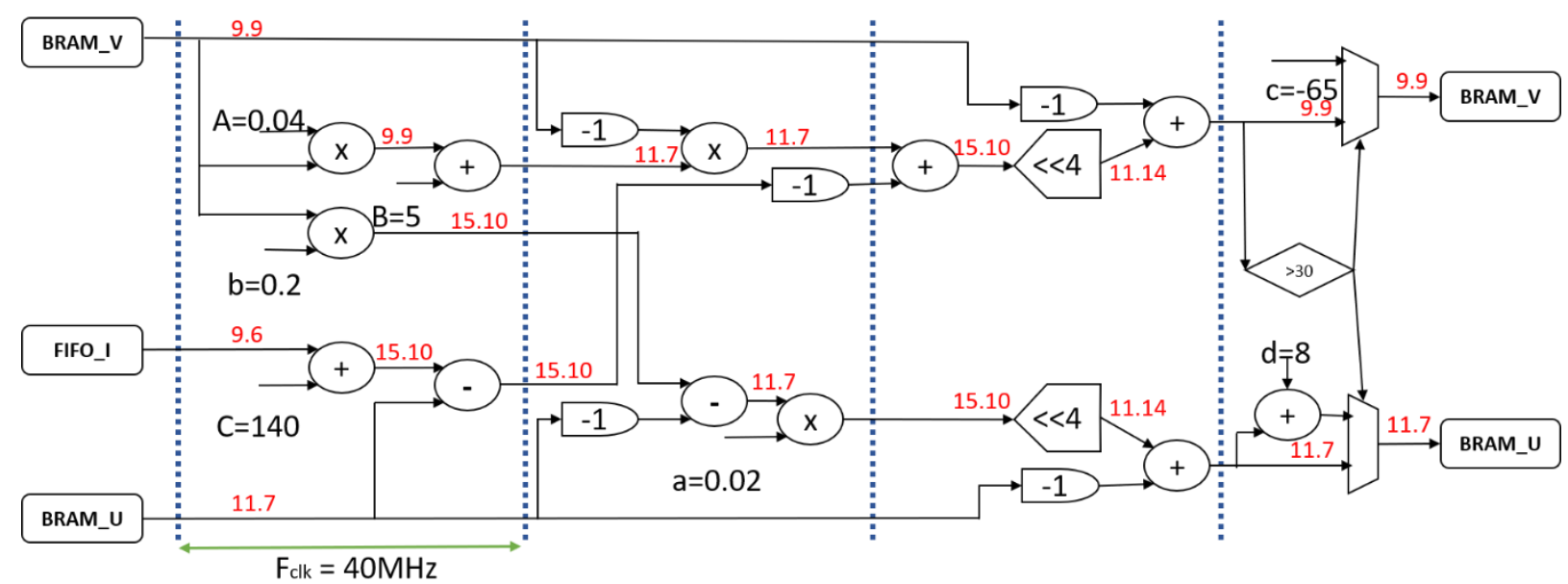

Figure 3 Izhikevich neuron architecture for FPGA 


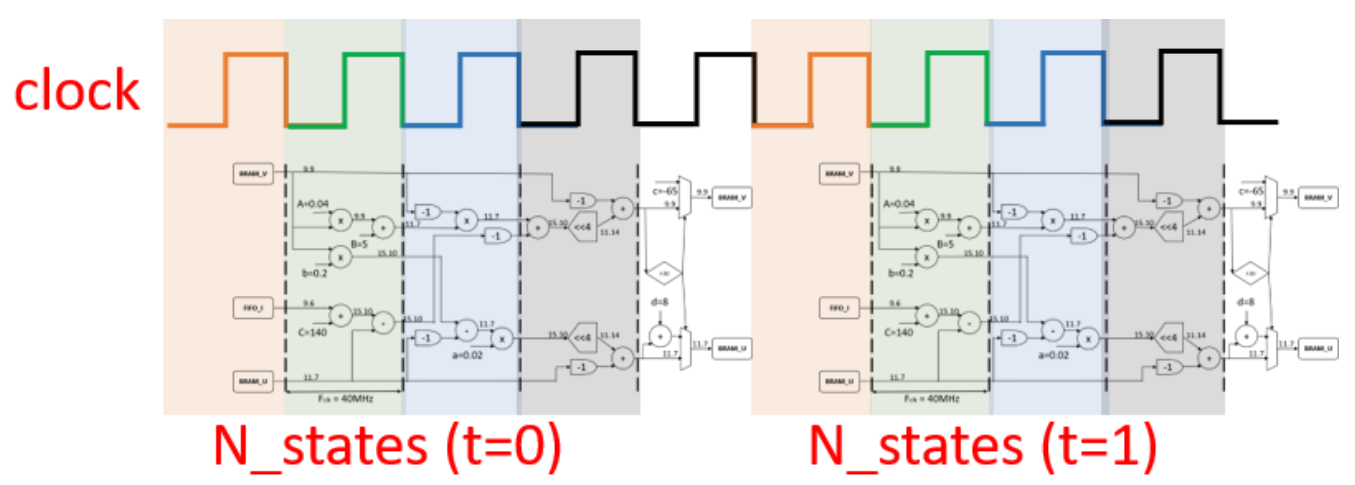

Figure 4 Timing Diagram representing the data flow through the proposed logical architecture for each clock cycle

\section{RESULTS}

The spike trains obtained by the Izhikevich neurons successfully encoded the stiffness of the manipulated materials. The implemented fast adapting neurons had higher spike rate for the stiffer materials during the loading phase.

Figure 5 shows the spike trains emulated by FPGA validated against the Izhikevich model simulated in Matlab using the codes provided in [25], changing the time step to $0.0625 \mathrm{~ms}$ to match the real-time execution of the system where the FPGA neuron was updated at $16 \mathrm{kHz}$. Both the neurons were stimulated with $\mathrm{I}=30 \mathrm{~mA}$ for $0.5 \mathrm{~s}$ and the spike timings were recorded as below. Furthermore, the resources utilization report of the above single-core neuron on Xilinx Spartan-6 1x45 of NI- sbRIO 9636 at 40MHz was as follows: Slice: $4.0 \%$; Slice LUTs: 7.9\%; Block Ram: 16.4\%; DSP48s: $10.3 \%$.

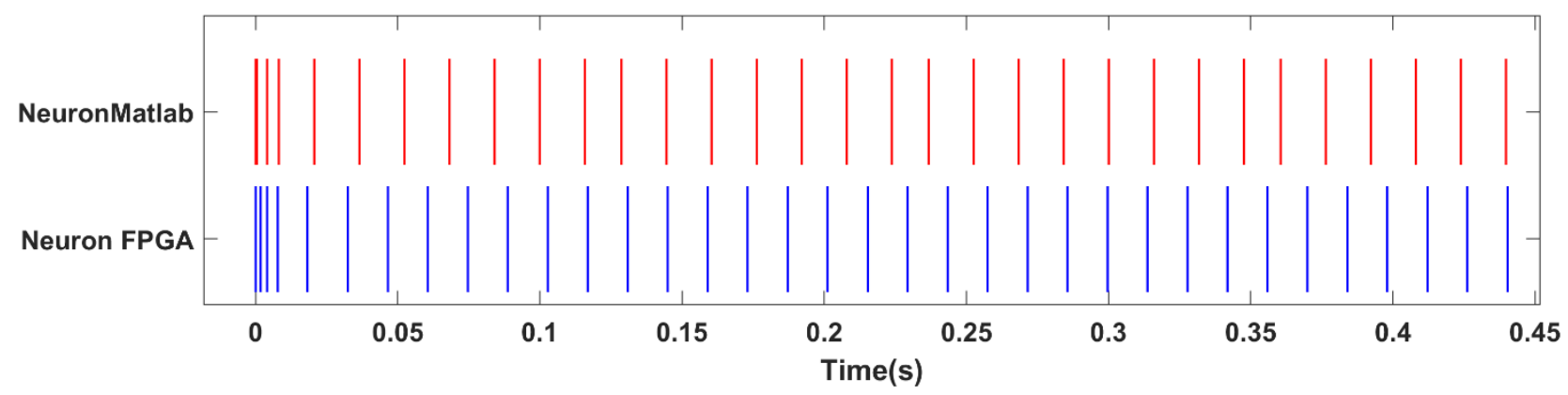

Figure 5 Matlab simulated Izhikevich spike (top in red) vs FPGA emulated spike trains (bottom in blue) for I = $30 \mathrm{~mA}$ updating membrane potential at $16 \mathrm{kHz}$ with an I update from $\mathrm{RT}$ at $4 \mathrm{~Hz}$.

Offline stiffness discrimination was performed on the grasping experimental dataset including four materials, and that was previously demonstrated to encode the stiffness of the material in [20] the slope of raw sensor data. By filtering we obtain the derivative of the slope which is directly related to the stiffness. This information encoded from the two selected FBGs is shown for the considered four materials in Figure 6. 

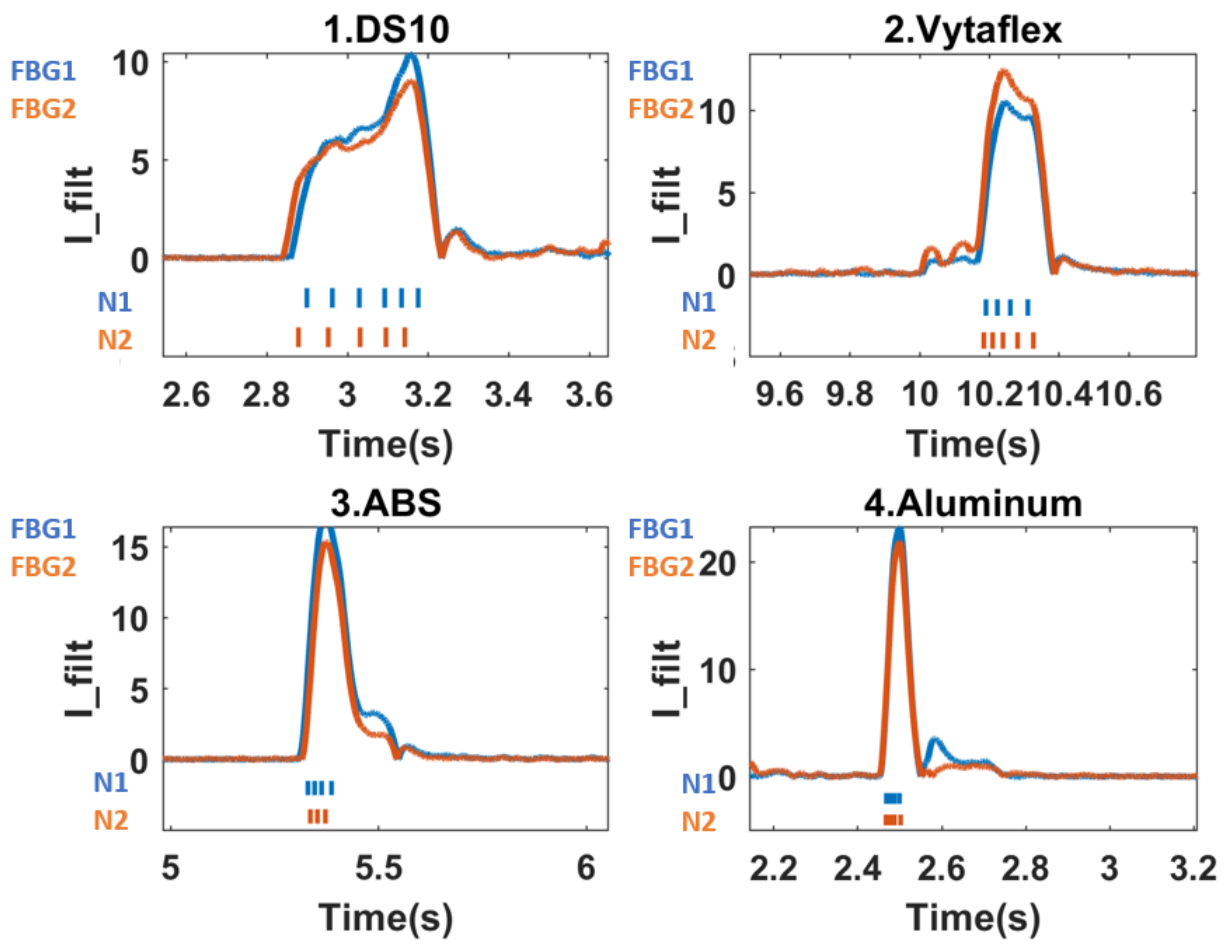

Figure 6 Windowed and filtered current $\left(I_{\text {filt }}\right)$ input to the neuron with corresponding spikes (N1 in blue corresponding to FBG1 while $\mathbf{N 2}$ in orange to FBG2) of the selected two FBGs (blue = FBG1 and red = FBG2) for the chosen four materials: (clockwise) DS10, Vytaflex, Aluminium and ABS

The stiffness information encoded into spikes was reconstructed (Figure 7) by measuring its temporal spacing in terms of inter-spike-intervals (ISI). The stiffer material, e.g. Aluminum that had a larger slope in the sensor data, produced faster spikes, hence a reduced ISI. Figure 7A shows the boxplot with the median as the central mark and the edges of the box representing the 25 th and 75 th percentiles, while the vertical line extends to the extreme datapoints and the outliers as ' + '. The larger number of outliers with ABS is due to the nonlinearity in the compressive material property at the chosen velocity of the grasp. An accuracy of $79.9 \%$ was obtained with a coarse tree classification performed on the obtained 562 samples of ISI in total, for all the four materials, with 50-fold cross validation (Figure 7B). 
A

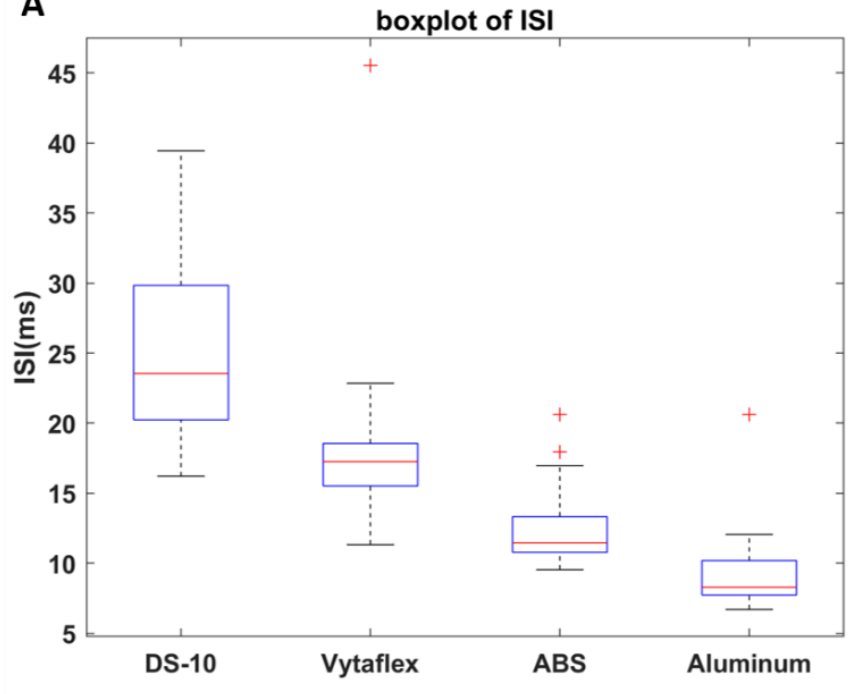

B

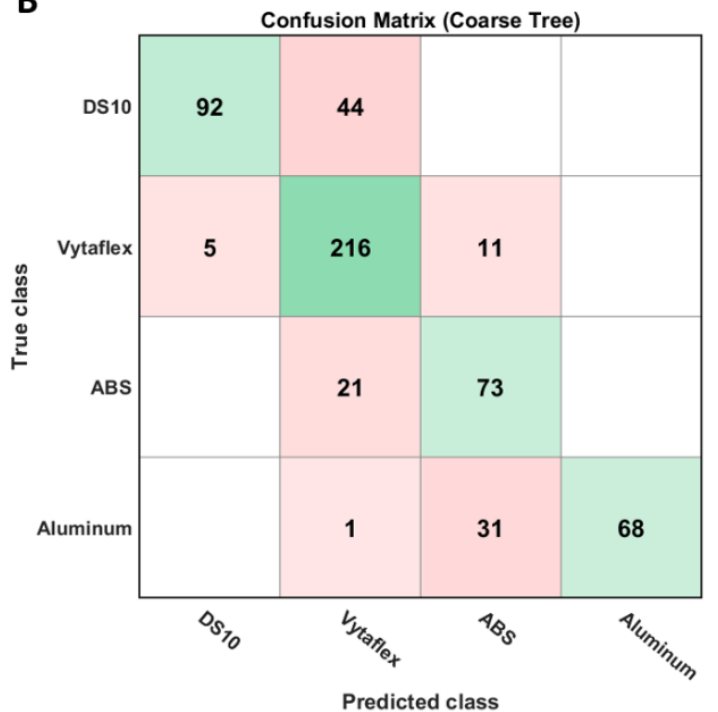

Figure 7 (A) Box-plot of inter-spike-interval of the four materials: The Young Modulus is increasing from left to right inturn increasing the spiking rate and decreasing the temporal space between the spikes; (B) Confusion matrix obtained from supervised classification with 50-folds cross validation and an accuracy of $\mathbf{7 9 . 9 \%}$

\section{DISCUSSION}

The results presented in this paper are promising in terms of neuromorphic encoding of stiffness information about the grasped objects during the loading phase with an accuracy of $79.9 \%$. The outputs of the FBGs were converted into spiking on/off events by means of a neuron model that emulates biological mechanoreceptors in encoding tactile information. This conversion, which was also implemented in a Field Programmable Gate Array, permits to reduce the bandwidth required by communication between sensing and computational subsystems of the robot. The developed tactile sensor was experimentally assessed through robotic manipulation tasks, in particular to discriminate the stiffness of grasped objects made of four different materials (Dragon Skin, Vytaflex, ABS and Aluminum). Based on the obtained results, the proposed encoding strategy permits to sense the sought material properties, thus fostering the potential of optic neuromorphic sensing in a range of applications, including neuro-prosthetics, neurorobotics and robotic telepresence.

This work will be extended for encoding other physical properties, like sliding information and object shape, and to further contribute to the control of the robotic arm based on the spiking tactile information. Biomimicry could be further improved by implementing a decoding algorithm based on the cuneate, second order, neuron architecture [30,31]. 


\section{BIBLIOGRAPHY}

1 Merolla PA, Arthur JV, Alvarez-Icaza R, Cassidy AS, Sawada J, Akopyan F, Jackson BL, Imam N, Guo C, Nakamura Y, et al. A million spiking-neuron integrated circuit with a scalable communication network and interface. Science. 2014 Aug;345(6197).

2 Akopyan F, Sawada J, Cassidy A, Alvarez-Icaza R, Arthur J, Merolla P, Imam N, Nakamura Y, Datta P, Nam G, et al. Truenorth: Design and tool flow of a $65 \mathrm{mw} 1 \mathrm{million}$ neuron programmable neurosynaptic chip. IEEE Transactions on Computer-Aided Design of Integrated Circuits and Systems.. 2015 Aug;34(10).

3 Lichtsteiner P, Posch C, Delbruck T. A 128 x128 120 dB 15 us Latency Asynchronous Temporal Contrast Vision Sensor. IEEE journal of solid-state circuits. 2008 Jan;43(2).

4 Hamilton TJ, Jin C, Van Schaik A, Tapson J. An active 2-D silicon cochlea. IEEE Transactions on biomedical circuits and systems. 2008 Apr;2(1).

5 Oddo CM, Raspopovic S, Artoni F, Mazzoni A, Spigler G, Petrini F, Giambattistelli F, Vecchio F, Miraglia F, Zollo L, et al. Intraneural stimulation elicits discrimination of textural features by artificial fingertip in intact and amputee humans. Elife. 2016 Mar;5.

6 Rongala UB, Mazzoni A, Oddo CM. Neuromorphic artificial touch for categorization of naturalistic textures. IEEE transactions on neural networks and learning systems. 2015 Sep;28(4).

7 Rongala UB, Mazzoni A, Camboni D, Carrozza MC, Oddo CM. Neuromorphic artificial sense of touch: bridging robotics and neuroscience. Robotics Research. 2018.

8 D’Abbraccio J, Massari L, Prasanna S, Baldini L, Sorgini F, Airò Farulla G, Bulletti A, Mazzoni M, Capineri L, Menciassi A, et al. Haptic Glove and Platform with Gestural Control For Neuromorphic Tactile Sensory Feedback In Medical Telepresence. Sensors. 2019 Jan;19(3).

9 Kandel ER, Schwartz JH, Jessell TM. Principles of neural science. New York: McGraw-Hill; 2000.

10 Noda K, Matsumoto K, Shimoyama I. Stretchable tri-axis force sensor using conductive liquid. Sensors and Actuators A: Physical. 2014.

11 Seminara L, Pinna L, Valle M, Basiricò L, Loi A, Cosseddu P, Bonfiglio A, Ascia A, Biso M, Ansaldo A, et al. Piezoelectric polymer transducer arrays for flexible tactile sensors. IEEE Sensors Journal,. 2013;13(10).

12 Townsend W. The BarrettHand Grasper-programmably Flexible Part Handling and Assembly. Industrial Robot: An International Journal. 2000;27(3).

13 Butterfaß J, Grebenstein M, Liu H, Hirzinger G. DLR-Hand II: Next generation of a dextrous robot hand. In: Proceedings 2001 ICRA. IEEE International Conference on Robotics and Automation; 2001 May. p. 109-114.

14 Ramos AM, Gravagne IA, Walker ID. Goldfinger: A non-anthropomorphic, dextrous robot hand. In Proceedings. In: IEEE International Conference on Robotics and Automation; 1999.

15 Yousef H, Boukallel M, Althoefer K. Tactile sensing for dexterous in-hand manipulation in robotics-A review.. Sensors and Actuators A: physical,. 2011;167(2). 
16 Karumanchi S, Edelberg K, Baldwin I, Nash J, Reid J, Bergh C, Leichty J, Carpenter K, Shekels M, Gildner M, et al. Team RoboSimian: semi-autonomous mobile manipulation at the 2015 DARPA robotics challenge finals. Journal of Field Robotics. 2017;32(2):305-32.

17 Johansson RS, Flanagan JR. Coding and use of tactile signals from the fingertips in object manipulation tasks. Nature Reviews Neuroscience. 2009 May;10(5).

18 Wolfbeis OS. Fiber-optic chemical sensors and biosensors. Analytical chemistry. 2004;76(12).

19 Begej S. Planar and finger-shaped optical tactile sensors for robotic applications.. IEEE Journal on Robotics and Automation. 1988. ;4(5).

20 Massari L, Oddo CM, Sinibaldi E, Detry R, Bowkett J, Carpenter KC. Tactile sensing and control of robotic manipulator integrating fiber Bragg grating strain-sensor. Frontiers in Neurorobotics. 2019;13(April):1-10.

21 Saccomandi P, Oddo CM, Zollo L, Formica D, Romeo RA, Massaroni C, Caponero MA, Vitiello N, Guglielmelli E, Silvestri S, et al. Feedforward neural network for force coding of an MRI-compatible tactile sensor array based on fiber Bragg grating.. Journal of Sensors. 2015.

22 Diamond ME, Von Heimendahl M, Knutsen PM, Kleinfeld D, Ahissar E. 'Where'and'what'in the whisker sensorimotor system. Nature Reviews Neuroscience. 2008.

23 Wan C,CG, Fu Y, Wang M, Matsuhisa N, Pan S, Pan L, Yang H, Wan Q, Zhu L, Chen X. An artificial sensory neuron with tactile perceptual learning. Advanced Materials. 2018;30(30).

24 Massaroni C, Saccomandi P, Schena E. Medical Smart Textiles Based on Fiber Optic Technology: An Overview. Journal of functional biomaterials. 2015 Arp;6(2).

25 E.M. I. Simple model of spiking neurons. IEEE Transactions on neural networks. 2003 Nov;14(6).

26 Heo JS, Chung JH, Lee JJ. Tactile sensor arrays using fiber Bragg grating sensors. Sensors and Actuators A: Physical. 2006 312-327.

27 Hudson KM, Condon M, Ackerley R, McGlone F, Olausson H, Macefield VG, Birznieks I. Effects of changing skin mechanics on the differential sensitivity to surface compliance by tactile afferents in the human finger pad. Journal of neurophysiology. 2015 Aug;114(4).

28 The MathWorks I. Train Decision Trees Using Classification Learner App. [Internet]. 19942019 [cited 2019]. Available from: https://www.mathworks.com/help/stats/train-decision-treesin-classification-learner-app.html.

29 Friedl MA, Brodley CE. Decision tree classification of land cover from remotely sensed data.. Remote sensing of environment. 1997;61(3).

30 Rongala UB, Mazzoni A, Chiurazzi M, Camboni D, Milazzo M, Massari L, Ciuti G, Roccella S, Dario P, Oddo CM. Tactile Decoding of Edge Orientation with Artificial Cuneate Neurons in Dynamic Conditions. Frontiers in Neurorobotics. 2019;13.

31 Rongala UB, Spanne A, Mazzoni A, Bengtsson F, Oddo C, Jörntell H. Intracellular dynamics in cuneate nucleus neurons support self-stabilizing learning of generalizable tactile representations. Frontiers in cellular neuroscience. 2018;12(p.120).

32 Indiveri G, Linares-Barranco B, Hamilton TJ, Van Schaik A, Etienne-Cummings R, Delbruck T, Liu SC, Dudek P, Häfliger P, Renaud S, et al. Neuromorphic silicon neuron circuits. Frontiers in neuroscience. 2011 May;5(73). 
33 Gunasekaran H, Spigler G, Mazzoni A, Cataldo E, Oddo CM. Convergence of regular spiking and intrinsically bursting Izhikevich neuron models as a function of discretization time with Euler method. Neurocomputing. 2019;350.

Proc. of SPIE Vol. 11136 1113608-10

Downloaded From: https://www.spiedigitallibrary.org/conference-proceedings-of-spie on 09 Sep 2019 Terms of Use: https://www.spiedigitallibrary.org/terms-of-use 